\title{
Impaled Nasopharyngeal Foreign Body
}

https://doi.org/10.47210/bjohns.2020.v28i3.289

Pritam Chatterjee, ${ }^{1}$ V D Prasanna Kumar Vasamsetty, ${ }^{1}$ Chiranjib Das, ${ }^{2}$ Sudip Kumar Das ${ }^{1}$

\begin{abstract}
Introduction
ABSTRACT

Foreign body cases are common in otolaryngologic practice, usually occurring in children. An impacted penetrating impaling foreign body can be one of the most challenging emergencies Management revolves around safe extraction of impaling object and prevention of complications. Here we share our experience with one such case of unusual foreign body impacted at an unusual site, with an extensive review of literature discussing management \& prevention of complications in similar cases.

Case Report

A 3 year old boy presented to us in ENT emergency with a long metallic hook impaled in nasopharynx for the past 8 hours. We were able to dislodge the impacted foreign body under direct endoscopic visualisation under general anaesthesia.

Discussion

Any such injury might be similar but no two injuries are the same. It needs combined expertise of multidisciplinary team usually consisting of ENT surgeon, anaesthesiologist, radiologist and may also need vascular surgeon and interventional radiologist at times. Several complications are reported in literature, knowledge of which would help preparedness and thus a better outcome.. $\underline{\text { Keywords }}$

Impalement; Foreign Bodies; Nasopharynx; Oropharynx
\end{abstract}

$\mathrm{F}$ oreign body cases are common in otolaryngologic practice, usually occurring in children. An impacted penetrating impaling foreign body can be one of the most challenging emergencies. Here we share our experience with one such case of unusual foreign body impacted at an unusual site.

\section{Case report}

A 3 years old boy presentedin ENT emergency with a long metallic hook struck somewhere in oral cavity for past 8 hours. The child was irritable with no signs of respiratory distress or any active bleed. X ray of soft tissue of neck (Figure 1) showed foreign body has probably not pierced any prevertebral or spinal structure but it has a hook at the impaling end which is lodged atnasopharynx.Flexible fibreoptic intubation was done. After elevating the soft palate using infant feeding tube, $2.7 \mathrm{~mm} 11 \mathrm{~cm} \mathrm{0}$-degree and $4 \mathrm{~mm} 17 \mathrm{~cm} 70$-degree endoscopes were used through nose and oral cavity to inspect actual site and mode of impaction. To rather surprise, there was no major injury. The hook passed from left side of oral cavity below right half of soft palate into the nasopharynx, entered through right choana, perforated lower part of posterior bony septum and appeared in the left nasal cavity.

Pulling the stick was definitely not working as it was lodged at the posterior border of palate\& perforated bony septum at an angulation. Pushing the hook back was also not working as it seemed the hook's diameter was larger than nasopharynx. With a better understanding of mode of impaction under direct visualisation, we were able to dislodge it by an upward rotational manoeuvre. Except for a small septal perforation and multiple minor lacerations on soft palate \& posterior pharyngeal wall, no other injuries were noted.Rest of the stay in hospital and follow-up was uneventful.

1 - Department of ENT, Bankura Sammilani Medical
College \& Hospital, Kolkata
2 - Department of ENT, Coochbehar Government Medical
College \& Hospital, Bankura
Corresponding author:
Dr V D Prasanna Kumar Vasamsetty
email: prasannavvd@gmail.com




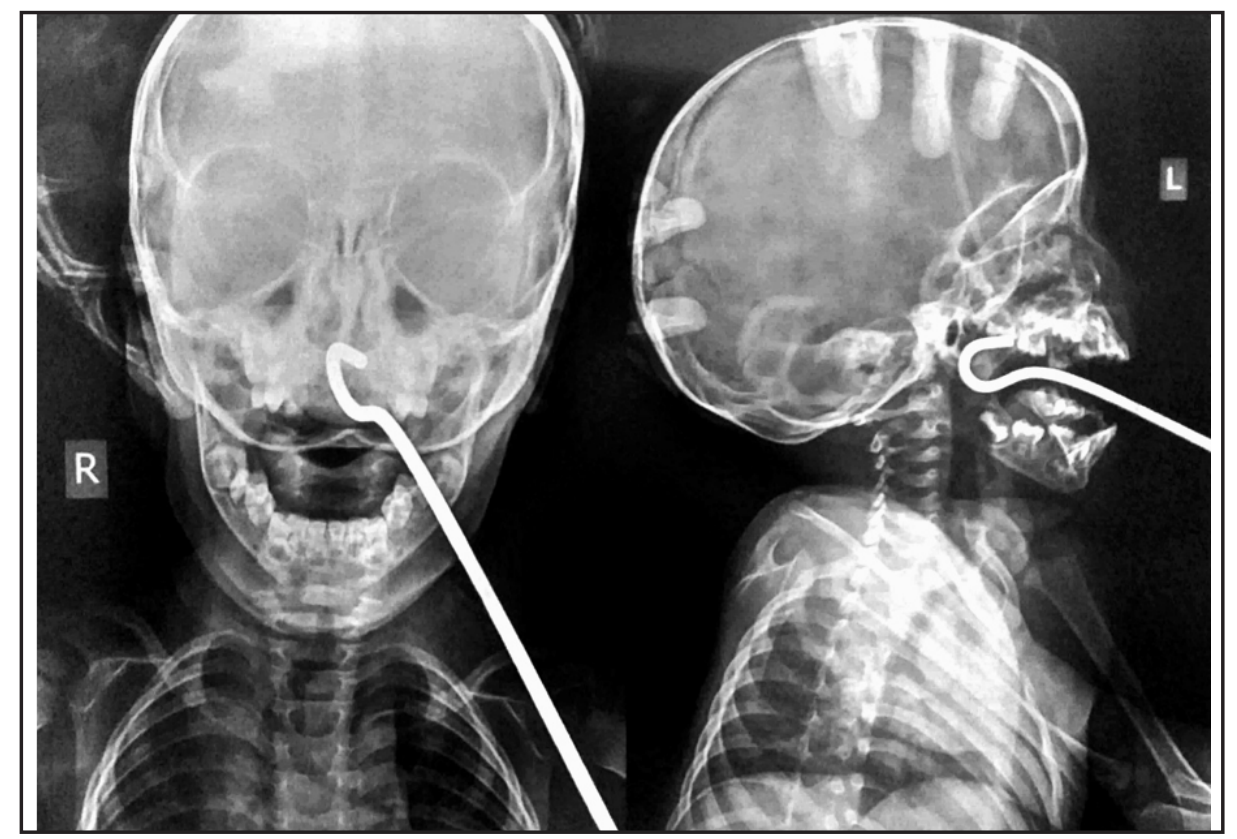

Fig. 1. X ray of soft tissue of neck showed the foreign body has probably not pierced or significantly injured any prevertebral or spinal structure but it has a hook which is lodged at the nasopharynx.

\section{Discussion}

Impalement injury to pharynx usually occur in children who fall prone while walking with foreign body in their mouths. ${ }^{1,2}$ The most frequently observed foreign bodies are toothbrushes and pencils.1,2Soft palate and tonsils are usually injured followed by hard palate, tongue, and posterior oropharynx. ${ }^{1,2}$ Nasopharynx is particularly rare area to be injured this way. ${ }^{3}$

The pharynx of a child is soft and stiff pointy foreign bodies can pass through posterior wall, injuring carotid artery or cervical spine. Management of these cases varies, which revolves around safe extraction of impaling object and prevention of complications. Impaling pharyngeal foreign body is a medical emergency. Delay of intervention can cause respiratory distress, due to edema or aspiration, impending vascular or spinal trauma\& infectious complications. . $^{3,4}$

At peripheral centres, where general physician or even if an ENT surgeon is available, but a proper diagnostic facility or a complete OT setup might not be available, it is always prudent to arrange for urgent referral to nearest tertiary care centre without any unnecessary delay. A detailed history ofinjury and object impacted is obtained, if possible, from an eye witness.

The initial assessment should focus on stabilizing potential life-threatening injuriesusing structured approach of advanced trauma life support (ATLS)1withsomecalculated risks which might fetch adequate time to reach higher centre.Immobilisation of patient \&impaling object is usually first essential step so that no further damage is caused.

Digital X-rays can be done for having general idea of shape \& size of impacted object, depth of penetration and site of impaction. It is always better to get, contrast enhanced CT and CT angiogram to rule out vascular injury or possible impaction on to major vascular channels. 1,33D reconstructed CT imaging are quite helpful for better understanding of orientation of foreign body with respect to surroundings and site of impaction. $\mathrm{CT}$ is also must for wooden or plastic foreignwith are not as radiopaque as metals.

If impending major vascular trauma is suspected, preoperative embolization can be done by interventional radiologist which can ensure safer extraction process. Bleeding can be severe, possibly from great vessel injury, requiring urgent repair by cardiovascular surgery 
team. If repair could not be performed, carotid artery ligation must be considered as a last resort. ${ }^{4}$

The potential of airway compromise may be apparent or predictable. In case of upper airway obstruction and severe intractable active bleed, one must plan to secure airway at the earliest. Anaesthesiologist can intubate, secure the airway and anaesthetise the patient for ENT surgeon to intervene. Other way round, in case of failed intubation, emergency tracheostomy can be can be performed by ENT surgeon to secure airway access and prevent aspiration.

Planning steps of extraction always needs individualised approach. It needs combined expertise of multidisciplinary team usually consistingof ENT surgeon, anaesthesiologist, radiologist and may also need vascular surgeon \& interventional radiologist. One of the important points toconsider is proper choice of instruments and operation room setup. Thisisparticularly important in case of sharp impacted pointedobjects like protruding hooks, blades, and open safety pins, which increase thedanger of perforation and vascular injury.

Anaesthesia was challenging in our case. Mouth opening was small and restricted. Preoxygenation was difficult as face mask couldn't be placed sufficiently in contact to face covering nose and mouth. All kinds of neck, head or chin manoeuvres needed for intubation were not done in fear of advancement of the foreign body and possible spinal trauma. Fibreoptic intubation was done considering all possibilities, keeping tracheostomy in hand.

Exact visualisation of site of impactionand damage is vital, but at times difficult due to shadowed anatomical location. It is difficult to immobiliseimpaling object in mouth. Luckily for our patient, with imaging, it was quite evident that it has not done any major damages and there were no major vessels at risk but surprisingly it has structed in such a way that it couldn't be taken out with ease. Never the less, in case an impending major vascular injury is suspected, before going in for actual extraction process, the vessel at risk should be secured for safer extraction process.

Wherever possible an endoscope can always be passed through nose and oral cavity to visualise oropharynx and nasopharynx. Controlled movement of the impacted object should be done under visualisation which eases extraction process just by mare better understanding of the situation. Direct exposure of the nasopharynx by retraction of soft palate with catheters could be helpful. ${ }^{5}$ $\mathrm{C}$-arm can also be used for a real time imaging to guide extraction.

After removal check endoscopy is to be done to identify any bleed and injury or residual broken piece of foreign body.For nasopharyngeal foreign bodies, nasal endoscopy provides the only method of proper visualization especially for sharp foreign bodies whose retrieval can also damage the surroundings. ${ }^{5}$

Immediate injury can range from minor laceration to major tear in posterior pharyngeal wall, soft palate, passervent ridge, pillars, injury to adenoid \& eustachian tube. ${ }^{6}$ Bleed may occur from minor mucosal lacerations and major arterial supplies in the area like sphenopalatine artery and tonsils,all kinds of which should be controlled by bipolar electrocauterization, sutures or packs as needed.

Any major laceration specially at palate level should be repaired with absorbable sutures to prevent late complications.6After removing foreign body, pharyngeal wound can be sutured or left open. It is better to leave wound open if it is infected, has slough or necrotic tissue. A clean wound should better be sutured. Leaving it open will unnecessarily subject it to very high bacterial load of oral secretions. Closing of an infected wound can lead to development of retropharyngeal abscess, ${ }^{6,7}$ sepsis $^{8}$ and even life-threatening mediastinitis, and for such wounds debridement and drainage is to be done. ${ }^{8}$

CT can be repeated for detection of residual foreign bodies suspected \& recurrent abscess patients. Magnetic resonance imaging has role in detection of neurological sequels, grisel syndrome and carotid artery pseudoaneurysm. ${ }^{9}$

Late complications may include velopharyngeal insufficiency or even stenosis. ${ }^{9,10}$ Smaller septal perforation usually heals spontaneously which happened for our case but there might be persistent bigger perforationscausing symptoms. Literature has also mentioned rare possibility of Grisel syndrome, caroticojugular fistulaandcarotid artery pseudoaneurysm. ${ }^{9}$

Neurological sequels due to thrombosis ${ }^{6,7}$ and stroke ${ }^{9}$ 
are potential severe complications. These isa high risk of air entering deep neck spaces causing widespread dissecting cervical emphysema, ${ }^{11}$ pneumothorax, ${ }^{11}$ pneumomediastinum, ${ }^{11}$ mediastinal emphysema ${ }^{8}$ or even pneumoencephalocoele. ${ }^{8}$ Edema of soft palate, tongue, retropharyngeal abscess \& dissecting emphysemas can present with stridor \& respiratory distress in postoperative period. Life threatening accident of carotid artery damage, ${ }^{11}$ shock and death have rarely been documented.

Innocuous injuries with minor soft-tissue trauma have also been associated with severe neurologic sequelae. ${ }^{12}$ Neurological examination may not correlate with development of symptoms, degree or mechanism of injury. ${ }^{12}$ The patient must be followed for at least 72 hours for early identification of possible lifethreatening complications. ${ }^{12}$ Appropriate antibiotics, anti-inflammatory and steroid have been found adequate in prevention and control of most of the complications. Oral feeding may be stopped for around 48 hours in suspected cases, for possibility of reoperation for tracheostomy or control of reactionary bleeds.

Neurovascular complications, despite their rarity have earned maximal discussion in literature. Traumatic lacerations and infectious complications have far more clinical relevance due to their frequency of occurrenceand should thus occupy a more prominent position in management flow chart. ${ }^{13}$

Any such injury might be similar but no two injuries are the same. From referral to extraction, each step should be considered with patience. One should always seek insights of experience, specialists from all required branches and well-equipped set up for a predictable positive outcome.

\section{References}

1. Hussain M (ed). ENT, Head \& Neck Emergencies: a Logan Turner companion. 2019; Taylor \& Francis Group, LLC. CRC Press

2. Tanaka T, Sudo M, Iwai K, Fujieda S, Saito H. Penetrating injury to the pharynx by a tooth brush in a paediatric patient: a case report. Auris Nasus Larynx 2002; 29(4):387-9

3. Diom ES, Diouf R, Diop el HM. Unusual nasopharyngeal foreign body: a hooked iron rod. Ear Nose Throat J. 2012; 91(7): E13-4

4. Kara İ, Ulutabanca H, Kökoğlu K, Güneş MS, Çağlı S. Pencil in the pharynx: Case report of a penetrating foreign body. Ulus Travma Acil Cerrahi Derg. 2016 ; 22(4):402-4

5. Kanotra S, Lateef M, Ahmed S. Nasopharyngeal Foreign Bodies. Indian Journal of Clinical Practice. 2012;22(8):392-6

6. Robert A. Belfer, Daniel W. Ochsenschlager, Sharon M. Tomaski. Penetrating injury to the oral cavity: A case report and review of the literature. The Journal of Emergency Med. 1995;13(3):331-5

7. Frantz R. Melio, Jonathan L. Jones, William T. Djang. Internal carotid artery thrombosis in a child secondary to intraoral trauma. The Journal of Emergency Med.1996; 14:429-33

8. Sagar S, Kumar N, Singhal M, Kumar S, Kumar A. A rare case of life-threatening penetrating oropharyngeal trauma caused by toothbrush in a child. J Indian Soc Pedod Prev Dent. 2010; 28:134-6

9. Steven M. Gottlieb, N. Paul Rosman, Robert A. Stich. Stroke in Children After Blunt Intraoral Trauma: Use of Magnetic Resonance Angiography as a Non-invasive Diagnostic Tool. J Child Neurol. 1996;11(5):419-22

10. Randall DA, Kang DR. Current management of penetrating injuries of the soft palate. Otolaryngol Head Neck Surg. 2006;135(3):356-60

11. Singer JI. Management strategy for penetrating oropharyngeal injury. Pediatr Emerg Care 1989;5(4):250-2

12. Radkowski D, McGill TJ, Healy GB, Jones DT. Penetrating trauma of the oropharynx in children. Laryngoscope 1993;103(9):991-4

13. Chauhan N, Guillemaud J, El-Hakim H. Two patterns of impalement injury to the oral cavity: Report of four cases and review of literature. Int J Pediatr Otorhinolaryngol. 2006; 70(8):1479-83. 